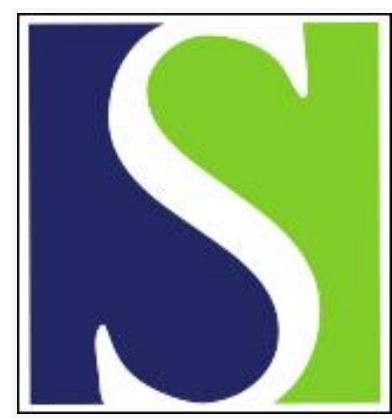

Scand J Work Environ Health 2004;30(5):410-420

https://doi.org/10.5271/sjweh.829

Issue date: Oct 2004

Prospective study of the relationship between musculoskeletal and psychological complaints and electromyographic activity during isometric muscular contractions in a working population

by Steingrímsdóttir ÓA, Knardahl S, Vøllestad NK

Affiliation: Department of Physiology, National Institute of Occupational Health, PO Box 8149 Dep, N-0033 Oslo, Norway. olofast@online.no

Key terms: electromyographic activity; isometric muscular contraction; muscular activity; musculoskeletal complaint; musculoskeletal pain; prospective study; psychological complaint; subjective complaint; working population

This article in PubMed: www.ncbi.nlm.nih.gov/pubmed/15529804 


\title{
Prospective study of the relationship between musculoskeletal and psychological complaints and electromyographic activity during isometric muscular contractions in a working population
}

\author{
by Ólöf Anna Steingrímsdóttir, MSc, ${ }^{1}$ Stein Knardahl, PhD, ${ }^{1,2}$ Nina Køpke Vøllestad, PhD ${ }^{3}$
}

\begin{abstract}
Steingrímsdóttir ÓA, Knardahl S, Vøllestad NK. Prospective study of the relationship between musculoskeletal and psychological complaints and electromyographic activity during isometric muscular contractions in a working population. Scand J Work Environ Health 2004;30(5):410-420.
\end{abstract}

\begin{abstract}
Objectives This study focused on determining whether musculoskeletal and psychological complaints reported monthly over a 4-month period predicted muscular activity during and immediately after standardized worktasks and whether muscular activity during and immediately after these tasks predicted changes in complaint severity in the following 12 months.

Methods Surface electromyography (EMG) was recorded bilaterally from the upper trapezius, middle deltoid, and forearm extensor muscles of 45 postal workers (30 women) during sustained submaximal (25\% of peak force) isometric contractions (wrist extension and shoulder abduction). Self-reported health complaints were recorded monthly. Musculoskeletal and psychological complaint-severity indices (MSI and PI, respectively) were computed from complaint-severity scores (intensity score $\times$ duration score). The history of complaints over the previous 4 months was included in adjusted regression models to predict muscular activity during and immediately after submaximal contractions. Muscular activity was included in adjusted models to predict changes in the complaint severity over the subsequent 12-month period.

Results A higher MSI predicted a lower EMG level in the trapezius muscle during submaximal contractions $(\mathrm{P}<0.014)$, whereas the PI did not predict the level of EMG in any of the muscles studied $(\mathrm{P}>0.194)$. The EMG activity did not predict changes in the complaint severity over the subsequent 12 months.

Conclusions These findings may support the hypotheses of pain adaptation or the dysfunction of synergistic muscular control in relation to musculoskeletal complaints. However, the findings did not indicate that increased or decreased muscular activity is a risk factor for heightened levels of complaints in the subsequent 12 months.
\end{abstract}

Key terms muscular activity, musculoskeletal, pain, psychological, subjective complaints.

The main objective of our study was to determine whether the severity of musculoskeletal or psychological complaints is related to muscular activity during standardized submaximal isometric work. The relations were studied in prospective designs.

Epidemiologic evidence suggests that static work is among the most common risk factors for musculoskeletal complaints $(1,2)$. The pathogenesis of musculoskeletal complaints is still largely unknown (3-7), although it is likely to involve a multifactor etiology that varies from person to person. It has been assumed that muscular pain and increased muscular activity or stiffness are related. [For definitions see the article by Simons \& Mense (8).] This view seems to stem from the hyperactivity model, which proposes that "pain is a consequence of a sustained spasm of skeletal muscle [p 422]" (9). Later models have proposed a self-perpetuating vicious circle in which pain and muscular activity or stiffness amplifies each other (10, 11). Lund and his co-workers (12) questioned vicious circle models and suggested the pain-adaptation model, in which reflex adaptations associated with pain involve decreased motoneuron output to nearby muscles that act as agonists and increased motoneuron activity to nearby antagonists. The pain adaptation may serve the purpose of immobilizing the painful, affected body part. According to the neuromuscular activation model, pain causes inhibition or delayed activation in specific muscles or muscle groups with key synergistic functions (13).

1 Department of Physiology, National Institute of Occupational Health, Oslo, Norway.

2 Department of Psychology, University of Oslo, Oslo, Norway.

3 Section for Health Science, University of Oslo, Oslo, Norway.

Reprint requests to: Ms Ólöf Anna Steingrímsdóttir, Department of Physiology, National Institute of Occupational Health, PO Box 8149 Dep, N-0033 Oslo, Norway. [E-mail: olofast@online.no] 
Building on the proposed mechanisms and on previously published epidemiologic evidence, we used a prospective design to investigate whether the average severity of musculoskeletal complaints over an extended period and muscular activity during and immediately after submaximal contractions are related. We chose to assess the activity of the trapezius, deltoid, and forearm extensor muscles by electromyography (EMG) to examine muscles of body regions with a high prevalence of complaints $(14,15)$. Furthermore, we were interested in investigating responses in a working population including workers with and without complaints. The participants were thus typical of those often used as controls in case-control studies.

From prospective studies on neck and back pain, Linton (16) concluded that anxiety, depression, distress, and related emotions are strongly associated with pain and disability. However, no link between psychological complaints and muscular activity has been established, even though experimental studies [eg, that of Wærsted \& Westgaard (17)] have found increased muscular activity in association with mental stressors. There is a shortage of studies on the relation between psychological factors and muscular activity during sustained submaximal contractions. We were interested in whether the heightened levels of muscular activity during cognitive tasks in sedentary work situations (17) also occur during submaximal contractions in workers with psychological complaints. In addition, we sought to determine whether muscular activity during and immediately after submaximal contractions predicts changes in the severity of psychological and musculoskeletal complaints over time. The postcontraction relaxation ability is thought to be important in the development of musculoskeletal disorders (18). In chronic pain patients, increased muscular activity has been observed [eg, by Elert et al $(19,20)$ and Fredin et al $(21)]$ during relaxation phases in dynamic activity, which also indicates a relation between complaints and the ability to relax the muscles.

Standardized sequences of muscular contractions allow more control than normal occupational settings over biomechanical challenges or other external conditions likely to cause variability in muscle responses. Hence we subjected workers to standardized laboratory sequences of shoulder abduction and wrist extension contractions until exhaustion. With this type of work, the initial phase may represent the response to the workload, and the final phase may represent the physiological response to fatigue. We therefore examined both the muscular activity in the first 40 seconds and final 5 seconds before exhaustion and how these measures of muscular activity related to complaints.

In designing this study, we asked the following two questions: (i) do musculoskeletal or psychological complaints during a 4-month period predict the subsequent endurance time of sustained submaximal contractions and muscular activity levels during and immediately after subsequent contractions and (ii) do the levels of muscular activity during or immediately after submaximal contractions predict changes in the severity of musculoskeletal or psychological complaints over the subsequent 12-month period?

\section{Study population and methods}

\section{Study design}

The study was part of a large prospective study on postal workers, in which data were collected over almost 3 years (22). The present data (figure 1) were obtained from one laboratory work session performed for each participant in the period July to September 1997 (force, endurance time, and EMG measurements) and monthly health-complaint reports collected over 16 consecutive months (4 months preceding and 12 months after the laboratory work session). The forms used for the healthcomplaint reports were distributed at the workplaces each month. They were mailed to those on long-term sick leave and to those who left the post offices during the study period. The Regional Ethical Committee for Medical Research approved the study.

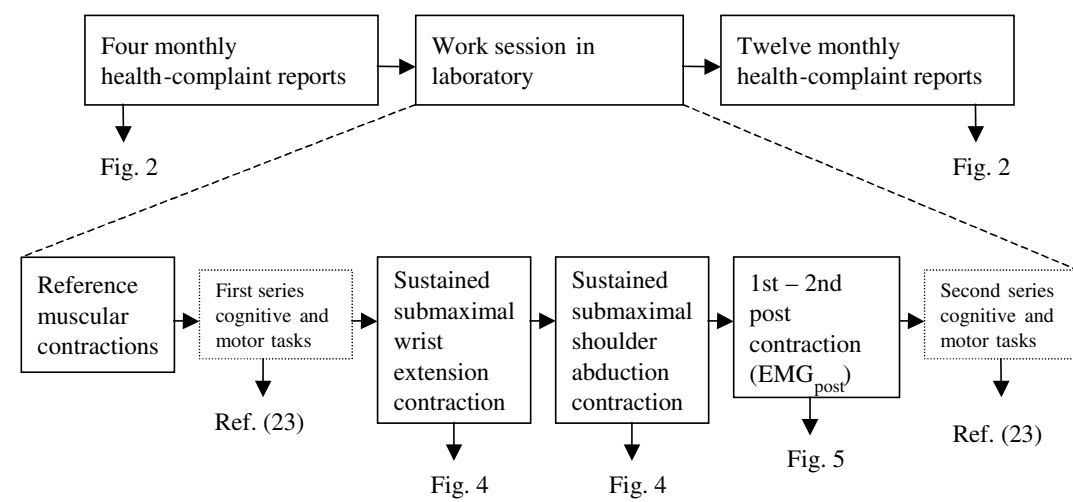

Figure 1. Flowchart of the categories used in the study. (EMG = electromyography) 


\section{Study population}

All counter staff in 11 post offices in Oslo were invited to participate in the main study. The inclusion criteria were permanent employment at a post office, working at least 19 hours a week, counter service as the main work function, and age less than 61 years. The exclusion criteria were any known systemic disease, pregnancy, or participant's self-reported inability to maintain a seated position for 3 hours. Experiments were conducted only when the workers had no vacation or sickness absence in the preceding week and had been at work for the preceding 3 days.

Written, informed consent was acquired from 104 subjects (67 women) of the 116 employees who met the inclusion criteria. During the interval between the worker inclusion and the laboratory experiment in 1997, some workers were excluded because of altered employment or work functions (28 persons), pregnancy (7 persons), sick leave certified by a physician (6 persons), or lower back pain ( 2 persons); 1 person also withdrew. Of the 60 remaining workers, we included 45 (30 women) who worked at the nine post offices nearest the laboratory. Eight persons (7 women) participating in the experiments dropped out during the following 12 months, six due to altered employment or work functions, one because of unease with the experiments, and one due to pregnancy.

The 30 women had a median age of 34 (range 2756) years, a median body weight of 66.5 (range 47-107) $\mathrm{kg}$, a median body height of 165 (range 157-180) cm,

Table 1. Items of the musculoskeletal complaint-severity index (MSI) and psychological complaint-severity index (PI). Each complaint-severity index (index range 0 to 9 ) was computed as the mean of the complaint-severity scores (score range 0 to 9 ). $C_{\alpha}$ represents the internal consistency of the indices (given as Cronbach's alpha).

\begin{tabular}{ll}
\hline Complaint-severity index & $\begin{array}{l}\text { Items with complaint-severity scores } \\
\text { (intensity score } \times \text { duration) }\end{array}$ \\
\hline $\mathrm{MSI}\left(\mathrm{C}_{\alpha}=0.75\right)$ & Headache \\
& Neck pain \\
& Pain in left shoulder or upper arm \\
& Pain in right shoulder or upper arm \\
& Pain in left forearm \\
& Pain in right forearm \\
& Pain in left wrist or hand \\
& Pain in right wrist or hand \\
& Pain in upper back \\
& Pain in lower back \\
& Chest pain \\
& Pain in lower extremities \\
& Sleep disturbances \\
& Fatigue \\
& Vertigo \\
& Depressed mood or depression \\
& Anxiety or restlessness \\
\hline
\end{tabular}

and a median body mass index (BMI) of 23.3 (range 16.9$34.5) \mathrm{kg} / \mathrm{m}^{2}$. For the $15 \mathrm{men}$, the respective medians were 34.5 (range 29-49) years, 80 (range 65-110) kg, 180 (range 170-190) cm, and 24.2 (range 20.1-34.7) kg/m².

\section{Monthly health-complaint reports}

At the end of each month, the participants rated the intensity and duration of 30 health complaints during the preceding 14 days. Each of the complaints was rated on a 4-point scale for intensity $(0=$ not troubled, $1=$ a little troubled, 2 = quite troubled, $3=$ seriously troubled) and a 3 -point scale for duration $(1=1-5$ days, $2=6-10$ days, $3=11-14$ days). A complaint-severity score was constructed for each of the complaints by multiplying the intensity score by the duration score, yielding a score range from 0 to 9 . Six compound complaint-severity indices were computed as a mean of different complaintseverity scores (index range 0 to 9 ), two of which we investigated in this study: a musculoskeletal complaintseverity index (MSI) and a psychological complaint-severity index (PI) (table 1).

The level of physical activity during the previous 14 days was rated on a 6-point scale. The participant rated exercise, sports, and activities that caused breathlessness ( $0=$ not at all, $1=0.5-1$ hours $/$ week, $2=1-2$ hours/ week, $3=2-3$ hours/week, $4=3-4$ hours/week, $5=4$ 5 hours/week, and $6=>5$ hours/week).

The intraindividual variability of complaint severity was large, as presented elsewhere (22). According to our findings (22), more than two reports are required when the aim is to obtain representative data for the averaging of complaints over time.

\section{Protocol}

The experiments started between 0800 and 0900 or 1200 and 1300 , depending on the participant's work schedule, and lasted 3 to 4 hours. At least 1 week before the day of the experiment, each participant was informed and trained to perform the shoulder-abduction and wristextension contractions. On the day of the experiment, the information and instructions were repeated.

The participant performed reference contractions, followed by a series of standardized cognitive and motor tasks, sustained submaximal wrist extension until exhaustion, sustained submaximal shoulder abduction until exhaustion, and a second series of the cognitive and motor tasks (figure 1). Muscular activity during the cognitive and motor task series has been described elsewhere (23).

Experimental work conditions. The room temperature during the experiments was 24 (SD 1) ${ }^{\circ} \mathrm{C}$. The participants were seated in an upright position, with their hips 
and knees flexed approximately 90 degrees and their ankles in a neutral position. The chair in which each participant sat was equipped with adjustable supports for the feet and lower back. During wrist extension, the chair was also equipped with adjustable supports for the arms. The experimenters adjusted the supports, and when comfortable to the participant, fixed the positions. All contractions were performed bilaterally.

Reference contractions. To determine reference values for the peak force and peak voluntary electrical activity $\left(\mathrm{EMG}_{\text {peak }}\right)$, we asked the participants to make three attempts at maximal muscular contraction, for both wrist extension and shoulder abduction. Each contraction lasted approximately 4 seconds, followed by 2 minutes of rest, during which the participants were told to relax. Experimenters gave verbal encouragement and visual force feedback to the participants to obtain contractions as near maximal as possible, and extra contractions were performed if the experimenters had any doubt about the quality of the first three contractions.

For the wrist extension measurements, both upper arms were held in a neutral position in the scapular plane with the elbows flexed at approximately 90 degrees and the forearms fully pronated and resting (proximal to the wrists) on the chair's arm supports. The participants pulled force transducers through wires that were attached vertically (approximately 90 degrees to the forearms) in fasteners on the cuffs distal to the wrists. During the contractions, the participants held their wrists steady in a neutral position, with their fingers relaxed. Contractions were used to obtain reference values for the forearm extensors.

For the shoulder abduction measurements, both upper arms were abducted 45 degrees in the scapular plane with the elbows flexed 90 degrees, the forearms semipronated, the wrists in a neutral position, and the fingers in a relaxed position. The participants pulled force transducers through wires that were attached diagonally (approximately 90 degrees to the upper arms) in fasteners on adjustable cuffs proximal to the elbow joints, ensuring 45-degree shoulder abduction. Contractions were used to obtain reference values for the upper trapezius and the middle deltoid muscles.

Submaximal isometric contractions. The participants were positioned as for the maximal contractions, and they then performed sustained bilateral wrist extension at $25 \%$ of their extension peak force. After 4 minutes of rest they performed sustained bilateral shoulder abduction at $25 \%$ of the abduction peak force. Visual feedback helped the participants maintain a steady force during the sustained isometric contractions. The participants were encouraged to maintain the contractions until exhaustion. Contractions were terminated simultaneously in both arms.

\section{Measurements and data acquisition}

The participants pulled force transducers (load cell 0 200 kg, Tokyo Sokki Kenkyujo Co., Ltd, Japan) connected by wires to adjustable cuffs. Bipolar surface EMG (type E-10-VS, Medicotest A/S, Ølstykke, Denmark, $20 \mathrm{~mm}$ inter-electrode distance) were recorded bilaterally from the upper trapezius muscle $(60 \%$ lateral to $\mathrm{C} 7$ on the line from $\mathrm{C} 7$ to the lateral edge of the acromion), the middle deltoid muscles (40\% distal to the acromion on the line from the lateral edge of the acromion to the muscular insertion), and the forearm extensors ( $25 \%$ distal to the radius head on the line from the radius head to the styloid process of the radius). Because of the known problems of EMG selectivity in forearm muscles, we investigated the responses in the extensors as a group of muscles instead of a single muscle.

The electrodes were taped onto shaved and cleaned (70\% ethanol) skin. Signals were amplified 216 times for the deltoid muscles, 448 times for the trapezius muscles, and 1009 times for the forearm muscles [Preamplifiers, Premed, Oslo, Norway; bandwidth of 10-3000 $\mathrm{Hz}$, common mode rejection ratio (CMRR) $>100 \mathrm{~dB}$, input impedance $>5 \mathrm{G} \Omega$ ]. The signals were then amplified twice.

To avoid possible alias signals (ie, external signal noise), a low-pass filter of $1000 \mathrm{~Hz}$ should be used in the recordings. Such a filter was not applied in 1997 , but only in later recordings $(10-1000 \mathrm{~Hz}$ band-pass filtering). Fast Fourier (FFT) analyses were undertaken for all the workers on the present EMG data and EMG data recorded in identical experiments in 1998. Two experienced researchers blinded for the recordings inspected and compared the data (in the bandwidth $0-500 \mathrm{~Hz}$ ). They saw no differences in signal content or quality.

Force and EMG recordings were converted (16-bit analogue-to-digital card, AT-MIO 16x, National Instruments, Austin, TX, USA) with a sampling frequency of $2000 \mathrm{~Hz}$ using a Labview system (National Instruments).

\section{Data analyses}

The peak force for wrist extension was determined as the highest of the three maximal isometric wrist extension contractions, and the peak force for shoulder abduction was similarly determined for the three maximal isometric shoulder abductions. For each contraction, peak force was determined as the highest average value over 0.1 second.

The raw EMG signals were corrected for baseline changes (direct current level) and were digitally bandpass filtered in the frequency range of $10-350 \mathrm{~Hz}$. The root mean square (rms) values of the signals were calculated over 0.1 second. To avoid artifacts caused by 
signals from the heart, we eliminated the rms-EMG amplitudes recorded simultaneously as the R in the QRScomplex, and the two closest EMG values. Noise levels were defined for each EMG channel as the lowest median rms-EMG amplitudes over 0.7 seconds, and then subtracted from all respective data.

The $\mathrm{EMG}_{\text {peak }}$ was defined for each muscle as the highest median rms-EMG amplitude over 0.7 seconds. In the analyses of rms-EMG during sustained submaximal contractions, the first and the last 2.5 -seconds of the contractions were omitted before further calculations. Then, the median rms-EMG values of the initial 40 seconds and the final 5 seconds of the contraction time were obtained, and they are subsequently referred to as $\mathrm{EMG}_{\text {sub40 }}$ and $\mathrm{EMG}_{\text {sub5 }}$. The $\mathrm{EMG}_{\text {post }}$ was calculated as the median rms-EMG from 1 to 2 seconds after the end of the submaximal contractions. All the EMG values during and immediately after the submaximal contractions are given relative to $\mathrm{EMG}_{\text {peak }}$.

\section{Statistical analysis}

The Statistical Package for the Social Sciences (SPSS 9.0 to 11.5 , Chicago, IL, USA) and the Confidence Interval Analysis (CIA 2.0, Trevor Bryant 2000, University of Southampton, Southampton, UK) were used for the analyses. The regression coefficients and the mean or median differences are reported with $95 \%$ confidence intervals $(95 \% \mathrm{CI})$. Other data are reported as means and standard deviations. The differences were considered significant at the $5 \%$ level.

Regression modeling. The contribution of the variables included in each regression model was determined through manual backward stepwise regression. After computing the crude estimates, we put all the independent variables into the full model and, through exclusion of the independent variables with the smallest and most insignificant contribution to the model (the largest Pvalues), selected the best subsets of the significant predictors. The final adjusted models were computed using variables with significant contributions $(\mathrm{P}<0.1$ used as an inclusion criteria in the adjusted models).

Prediction of endurance time and muscular activity during and immediately after the contractions. The dependent variables of the various multiple regression models were the endurance time of the submaximal contractions and the $\mathrm{EMG}_{\text {sub40 }}, \mathrm{EMG}_{\text {sub5} 5}$, and $\mathrm{EMG}_{\text {post }}$.

The independent variables were the health complaints (MSI and PI) during the 4 months preceding the laboratory experiment, age, gender $($ women $=0$, men $=$ 1 ), and the level of physical activity (scale 0 to 6 ). The effects of the interaction between the physical activity level and the complaint variable, the interaction between age and the complaint variable, and the interaction between the MSI and the PI in the full models were examined. Analyses were performed independently for each of the three muscles.

In addition, we used similar regression models to examine whether the presence of complaints (mean of last 4 months; categorized as 0 if no complaints, and 1 if any complaints) in the neck, the ipsilateral shoulder or upper arm or in the ipsilateral forearm, wrist and hand predicted the $\mathrm{EMG}_{\text {sub40 }}$ or $\mathrm{EMG}_{\text {sub5 }}$ in the trapezius (during abduction) or forearm extensors (during extension). The analyses were performed independently for each muscle and each complaint.

Prediction of changes in complaint severity over a 12month period. The dependent complaint variables were either the MSI or the PI over the 12-month period following the laboratory experiments (mean of 12 months) and of the mean of the 2 months with highest complaint severity within that 12 -month period. The changes in MSI or PI were examined, with the inclusion of the MSI or PI over the preceding 4 months as independent variables. The other independent variables of the regression models were $\mathrm{EMG}_{\text {sub40 }}, \mathrm{EMG}_{\text {sub5, }}$ and $\mathrm{EMG}_{\text {post. }}$ These were calculated, as already described, and adjusted for age, gender, and the level of physical activity during the previous 4 months. We examined the effects of the interaction between physical activity and the EMG levels, and the interaction between age and the EMG levels in the full models. The effect of the EMG of each of the three muscles on the changes in the MSI and PI were investigated separately.

\section{Results}

\section{Complaint severity}

The medians of the musculoskeletal and psychological complaint-severity indices ranged between 0.14 and 0.20 during the 4 months before and the 12 months after the laboratory experiments (figure 2). Some of the workers appeared to be more afflicted than others, exhibiting scores between 2 and 3 on the 0 to 9 scale. None of the indices differed statistically significantly between the men and women $(\mathrm{P}>0.26)$.

\section{Maximal and submaximal contractions}

The mean peak force of the maximal contractions was 147 (SD 49) $\mathrm{N}$ for wrist extension and 173 (SD 55) $\mathrm{N}$ for shoulder abduction. The mean $\mathrm{EMG}_{\text {peak }}$ was 454 (SD 212) $\mu \mathrm{V}$ for the forearm extensors, 933 (SD 358) $\mu \mathrm{V}$ for the trapezius, and 573 (SD 266) $\mu \mathrm{V}$ for the deltoid muscle. 
The endurance time of the wrist extension and shoulder abduction was 183 (SD 69) and 165 (SD 88) seconds, respectively. The average forces during the contractions were slightly higher than targeted, 28 (SD 2)\% for both the wrist extension and the shoulder abduction. The relative force was similar for the men and women during the wrist extension $(\mathrm{P}=0.99)$, and the endurance times did not differ significantly $(\mathrm{P}=0.38)$. During the shoulder abduction, however, the women worked with slightly higher relative force than the men (mean difference $2.4 \%, \mathrm{P}=0.03,95 \%$ CI $0.1-2.7$ ), and the men had longer endurance times than the women [women 146 (SD 75) seconds, men 205 (SD 101) seconds, $\mathrm{P}=0.03$ ].

Typical EMG recordings are shown for one participant in figure 3. Nearly identical EMG responses were recorded from the left and right sides of the body (Røe et al, submitted). Therefore, only the analyses of the data from the left side are presented.

During the first 40 seconds of submaximal wrist extension, the mean EMG level of the forearm extensors was 25 (SD 8)\% of the $\mathrm{EMG}_{\text {peak }}\left(\mathrm{EMG}_{\text {sub40 }}\right.$ in figure $\left.4 \mathrm{~A}\right)$. The EMG level was higher in the final 5 seconds, reaching 39 (SD 12)\% of the $\mathrm{EMG}_{\text {peak }}\left(\mathrm{EMG}_{\text {sub5 }}\right.$ in figure $\left.4 \mathrm{~B}\right)$. There was no difference between the men and women in the activity of the extensors $(\mathrm{P}>0.14)$. The mean EMG levels of the trapezius and deltoid muscles during wrist extension were less than $5 \%$ of the $\mathrm{EMG}_{\text {peak }}$.

During submaximal shoulder abduction, the $\mathrm{EMG}_{\text {sub40 }}$ values were 21 (SD 7) and 23 (SD 9)\% of the $\mathrm{EMG}_{\text {peak }}$ (figure 4A) for the trapezius and deltoid muscles, respectively. For the trapezius muscle, a slightly higher EMG value was observed in the final 5 seconds, reaching 32 (SD 13)\% of the $\mathrm{EMG}_{\text {peak }}\left(\mathrm{EMG}_{\text {sub5 }}\right.$ in figure $\left.4 \mathrm{~B}\right)$. The deltoid muscle showed about the same value in the final 5 seconds $\left[\mathrm{EMG}_{\text {subs }} 23\right.$ (SD 11)\% of the $\left.\mathrm{EMG}_{\text {peak }}\right]$,

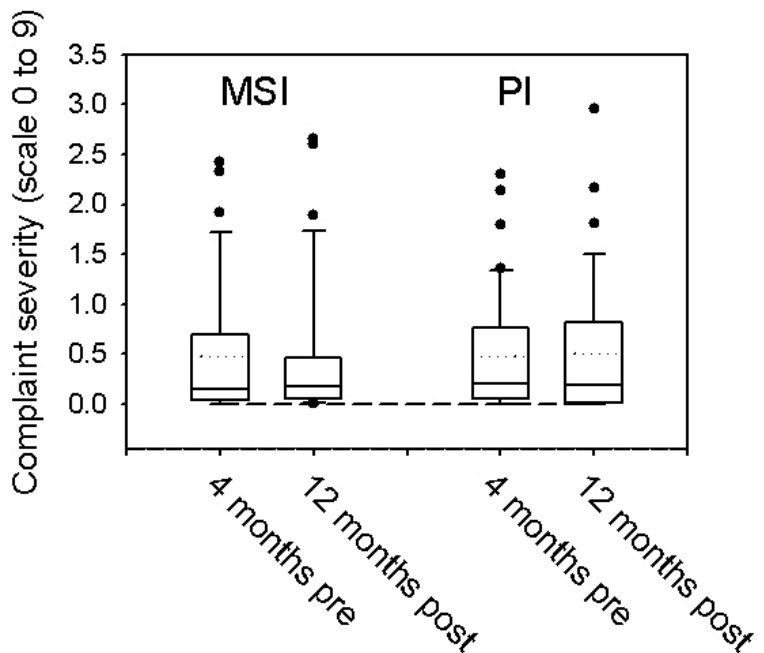

Figure 2. Box plot of the musculoskeletal complaint-severity index (MSI) and the psychological complaint-severity index (PI) for the 4 monthly reports preceding the laboratory experiment (4 months pre; $\mathrm{N}=45$ ) and the following 12 monthly reports (12 months post; $\mathrm{N}=37$ ). The boxes represent the 25th to 75 th percentiles, the solid lines within the boxes are the median values, the dotted lines within the boxes are the mean values, the whiskers are the 10th and 90th percentiles, and the points represent outliers. The broken line represents 0 .

as during the first 40 seconds. We observed no differences between the men and women for the trapezius $(\mathrm{P}>0.12)$ or deltoid $(\mathrm{P}=0.98)$ muscles in the final $5 \mathrm{sec}-$ onds although the women exhibited a higher mean $\mathrm{EMG}_{\text {sub40 }}$ for the deltoid muscles (P=0.028). The EMG values of the forearm extensors during shoulder abduction were about $3 \%$ of the $\mathrm{EMG}_{\text {peak }}$.

\section{Postcontraction muscular activity}

The mean $\mathrm{EMG}_{\text {post }}$ of the forearm extensors was 5.6 (SD $5.9) \%$ of the $\mathrm{EMG}_{\text {peak }}$ during the 2nd second after the
Wrist extension
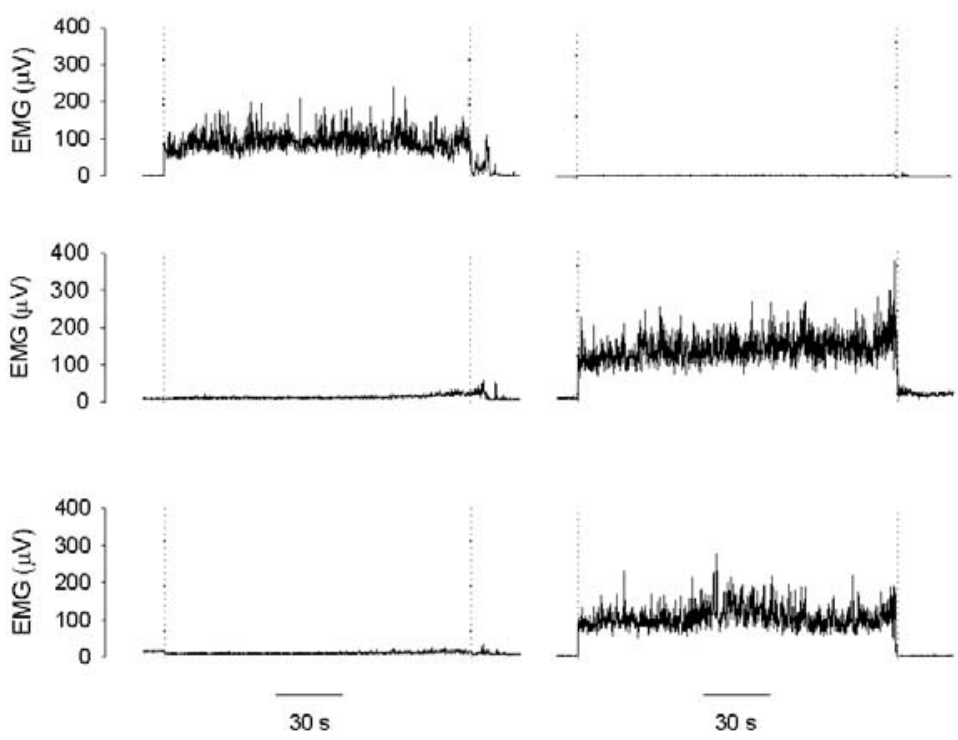

Upper trapezius

Forearm

extensors

Middle deltoid
Figure 3. Root mean square-electromyography (rms-EMG) of the left forearm extensors, left upper trapezius, and left middle deltoid muscles during sustained submaximal isometric wrist extension and sustained submaximal isometric shoulder abduction contractions. EMG signals from one participant are shown. The vertical dotted lines represent the beginning and end of the contractions. See the methods section for more details. 

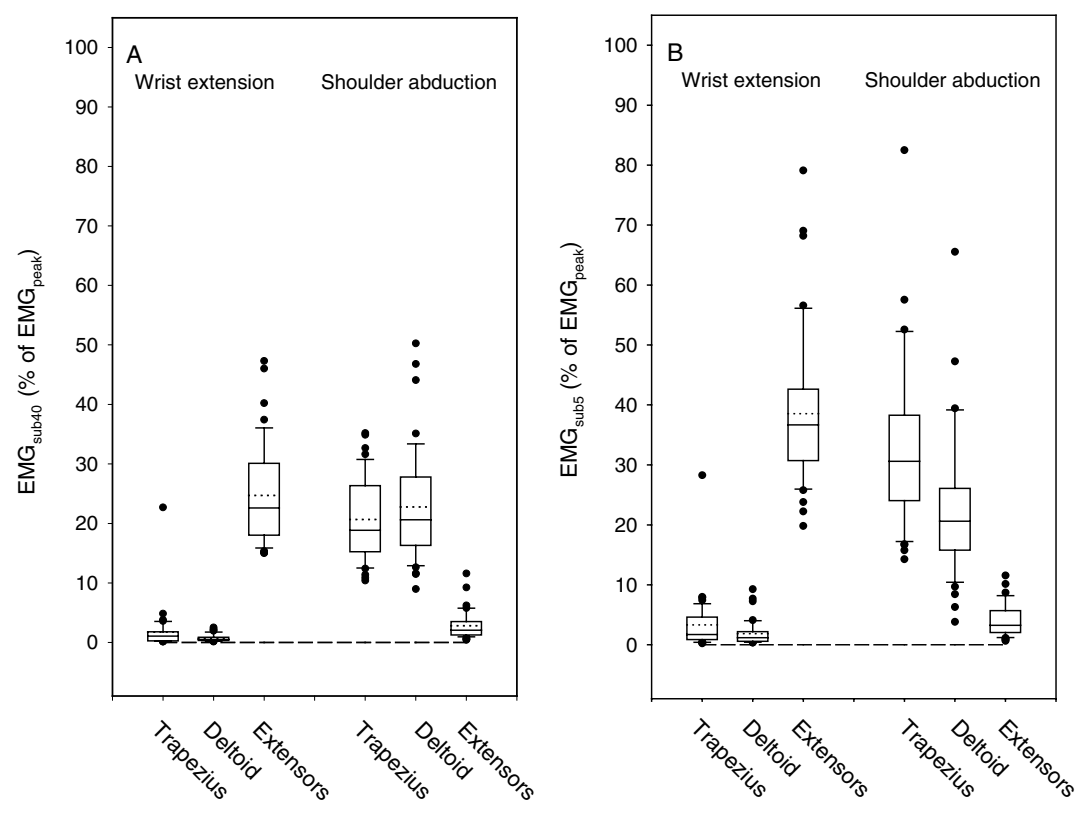

Figure 4. Box plot of the electromyographic (EMG) levels (as percentage of $E M G_{\text {peak }}$ ) during the first 40 seconds $(A)$ and the last 5 seconds (B) of the sustained submaximal contractions during the wrist extension $(\mathrm{N}=45)$ and shoulder abduction ( $\mathrm{N}=44)$. The EMG values for the left upper trapezius, left middle deltoid, and left forearm extensor muscles are shown. The boxes represent the 25th to 75th percentiles, the solid lines within the boxes show the median values, the dotted lines within the boxes represent the mean values, the whiskers are the 10th and 90th percentiles, and the points represent outliers. The broken line represents $0 .\left(E M G_{\text {peak }}=\right.$ peak voluntary electrical activity, $\mathrm{EMG}_{\mathrm{sub} 40}=\mathrm{EMG}$ during the first 40 seconds of submaximal contraction, $\mathrm{EMG}_{\text {sub5 }}=$ EMG during the last 5 seconds of submaximal contraction)

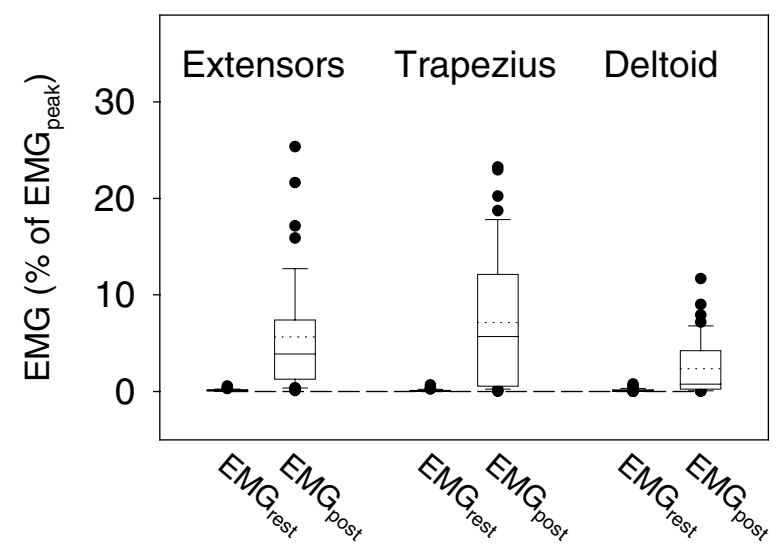

Figure 5. Box plot of the electromyographic (EMG) levels (as the percentage of $E M G_{\text {peak }}$ ) at rest and during the 2nd second after completed sustained submaximal contractions in the left forearm extensors, left upper trapezius, and left middle deltoid muscles $(\mathrm{N}=45$ in extension and $\mathrm{N}=44$ in abduction). Data for the forearm extensors were obtained after wrist extension, data for the trapezius and deltoid muscles were obtained after shoulder abduction. The boxes represent the 25th to 75th percentiles, the solid lines within the boxes show the median values, the dotted lines within the boxes represent the mean values, the whiskers are the 10th and 90th percentiles, and the points represent outliers. The broken line represents $0 .\left(E M G_{\text {peak }}=\right.$ peak voluntary electrical activity, $\mathrm{EMG}_{\text {rest }}=$ lowest EMG 0.1 -second values at rest, $E M G_{\text {post }}=E M G$ values 1st to 2 nd second postcontraction)

end of the submaximal extension. The level was 7.1 (SD 7.1)\% of the $\mathrm{EMG}_{\text {peak }}$ in the trapezius muscle and 2.4 (SD $3.0) \%$ of the $\mathrm{EMG}_{\text {peak }}$ in the deltoid muscle (figure 5).

\section{Predictors of endurance time and muscular activity during and immediately after submaximal contractions}

A higher level of physical activity in the previous months predicted a longer endurance time for shoulder abduction $(\beta=19.9, \quad P=0.047,95 \%$ CI $0.3-40)$ but not for wrist extension $(\mathrm{P}=0.79)$. Neither the MSI nor the PI predicted the endurance time or the $\mathrm{EMG}_{\text {post }}(\mathrm{P}>0.09)$.

The crude estimates of the relations between complaint severity and muscular activity during the submaximal contractions were not statistically significant. However, after adjustment for the level of physical activity (PA) and an interaction between the MSI and the level of physical activity (MSI $\times$ PA), a higher level of musculoskeletal complaint severity predicted a lower $\mathrm{EMG}_{\text {sub40 }}$ in the trapezius muscle during wrist extension (MSI: $\beta=-4.11, P=0.005,95 \%$ CI $-6.9--1.3$; PA: $\beta=-0.73, P=0.061,95 \%$ CI $-1.5--0.0$; MSI $\times$ PA: $\beta=1.95, P<0.001,95 \%$ CI $1.1-2.8$ ), whereas the psychological complaint severity did not. Neither musculoskeletal nor psychological complaint severity was a statistically significant predictor of the muscular activity level of the forearm extensors or the deltoid muscle during the first 40 seconds of wrist extension $(\mathrm{P}>0.09)$.

More severe musculoskeletal complaints also predicted a lower EMG level in the trapezius muscle during the first 40 seconds of shoulder abduction (table 2). However, the severity of musculoskeletal or psychological complaints did not predict the EMG levels of the deltoid muscle or of the forearm extensors during the abduction $(\mathrm{P}>0.10)$.

To examine whether muscular activity at the end of the contraction was predicted by the severity of complaints, we performed the same analyses with $\mathrm{EMG}_{\text {sub5 }}$ as the dependent variable. The MSI in the previous 4 months predicted a lower EMG in the upper trapezius muscle $(\mathrm{P}=0.04)$ in the final phase of shoulder abduction. 
The analyses showed no other statistically significant predictions ( $\mathrm{P}>0.14)$.

Because the MSI is calculated as an average of musculoskeletal complaints from the entire body, it is of importance to examine whether similar associations were found between pain and the $E M G_{\text {sub } 40}$ determined for the same area. The presence of pain in the shoulder and upper arm during the preceding 4 months predicted a significantly lower $\mathrm{EMG}_{\text {sub40 }}$ for the trapezius muscle during shoulder abduction (table 3$)$ and wrist extension $(\mathrm{P}=0.03)$. No other statistically significant predictive effects on $\mathrm{EMG}_{\text {sub40 }}$ were found $(\mathrm{P}>0.24)$.

Table 2. Prediction of the electromyographic (EMG) values, as percentage of peak voluntary electrical activity $\left(E M G_{\text {peak }}\right)$, in the left upper trapezius during the first 40 seconds of sustained submaximal shoulder abduction $\left(\mathrm{EMG}_{\text {sub40 }}\right)$. The musculoskeletal complaint-severity index (MSI) and psychological complaint-severity index (PI) from the previous 4 monthly reports were included as the main predictors in the multiple regression model. The main predictors were adjusted for gender, age, physical activity (PA) during the previous 4 months, the interaction between the MSI and the PI, the interaction between the MSI and physical activity, and the interaction between the PI and physical activity.

\begin{tabular}{|c|c|c|c|c|c|c|}
\hline \multirow[t]{3}{*}{$\begin{array}{l}\text { Independent } \\
\text { variables }\end{array}$} & \multicolumn{6}{|c|}{$\begin{array}{l}\mathrm{EMG}_{\text {sub40 }}\left(\% \text { of } E M G_{\text {peak }}\right) \text { in the left upper } \\
\text { trapezius during shoulder abduction }\end{array}$} \\
\hline & \multicolumn{3}{|c|}{ Crude estimates } & \multicolumn{3}{|c|}{ Adjusted estimates } \\
\hline & $\beta$ & P-value & $95 \% \mathrm{Cl}$ & $\beta$ & P-value & $95 \% \mathrm{Cl}$ \\
\hline MSI & -0.97 & 0.549 & $-4.2-2.3$ & -9.09 & $0.015^{\mathrm{a}}$ & $-16.3--1.9$ \\
\hline PI & -0.14 & 0.937 & $-3.7-3.4$ & . & . & . \\
\hline Gender & -3.43 & 0.123 & $-7.8-1.0$ & . & . & . \\
\hline Age & -0.02 & 0.905 & $-0.3-0.3$ & . & $\cdot$ & $\cdot$ \\
\hline PA & -1.04 & 0.202 & $-2.6-0.6$ & -2.74 & $0.011^{\mathrm{a}}$ & $-4.8--0.7$ \\
\hline MSI × PA & . & . & $\cdot$ & 2.81 & 0.009 a & $0.8-5.0$ \\
\hline
\end{tabular}

a $P<0.05, N=44$.

\section{Muscular activity as a predictor of changes in complaint severity over a 12-month period}

None of the measures of muscular activity $\left(\mathrm{EMG}_{\mathrm{sub} 40}\right.$, $\mathrm{EMG}_{\text {sub5}}, \mathrm{EMG}_{\text {post }}$ ) predicted changes in the severity of musculoskeletal or psychological complaints over a 12month period $(\mathrm{P}>0.10)$ or when the mean of the 2 months with the highest complaint severity was used $(\mathrm{P}>0.06)$. Table 4 shows the results of the analysis to

Table 3. Prediction of the electromyographic (EMG) values, as the percentage of peak voluntary electrical activity $\left(E M G_{\text {peak }}\right)$, in the left upper trapezius during the first 40 seconds of sustained submaximal shoulder abduction $\left(\mathrm{EMG}_{\text {sub40 }}\right)$. Pain in the ipsilateral shoulder or upper arm and pain in the ipsilateral forearm, wrist and hand from the previous 4 monthly reports (categorized as 0 if no complaints and 1 if any complaints) were included as the main predictors in the multiple regression models. The main predictors were adjusted for gender, age, physical activity (PA) during the previous 4 months, and the interaction between the complaint variables and physical activity.

\begin{tabular}{|c|c|c|c|c|c|c|}
\hline \multirow[t]{3}{*}{$\begin{array}{l}\text { Independent } \\
\text { variables }\end{array}$} & \multicolumn{6}{|c|}{$\begin{array}{l}E M G_{\text {sub40 }}\left(\% \text { of } E M G_{\text {peak }}\right) \text { in the left upper } \\
\text { trapezius during shoulder abduction }\end{array}$} \\
\hline & \multicolumn{3}{|c|}{ Crude estimates } & \multicolumn{3}{|c|}{ Adjusted estimates } \\
\hline & $\beta$ & P-value & $95 \% \mathrm{Cl}$ & $\beta$ & P-value & $95 \% \mathrm{Cl}$ \\
\hline $\begin{array}{l}\text { Pain in shoulder } \\
\text { or upper arm }\end{array}$ & -3.24 & 0.138 & $-7.6-1.1$ & -9.44 & $0.015^{a}$ & $-17.0--1.9$ \\
\hline Gender & -3.43 & 0.123 & $-7.8-1.0$ & . & . & . \\
\hline Age & 0.02 & 0.905 & $-0.3-0.3$ & • & $\cdot$ & $\cdot$ \\
\hline PA & -1.04 & 0.202 & $-2.7-0.6$ & -2.45 & $0.024^{a}$ & $-4.6--0.3$ \\
\hline $\begin{array}{l}\text { Pain shoulder or } \\
\text { upper arm } \times \text { PA }\end{array}$ & $\cdot$ & $\cdot$ & $\cdot$ & 3.07 & 0.051 & $-0.01-6.2$ \\
\hline $\begin{array}{l}\text { Pain in forearm, } \\
\text { wrist and hand }\end{array}$ & -1.03 & 0.671 & $-6.0-3.8$ & -11.2 & $0.008^{a}$ & $-19.4--3.0$ \\
\hline Gender & -3.43 & 0.123 & $-7.8-1.0$ & . & . & . \\
\hline Age & 0.02 & 0.905 & $-0.3-0.3$ & $\cdot$ & $\cdot$ & $\cdot$ \\
\hline PA & -1.04 & 0.202 & $-2.7-0.6$ & -2.83 & $0.005^{a}$ & $-4.7--0.9$ \\
\hline $\begin{array}{l}\text { Pain forearm, } \\
\text { wrist and hand } x \\
\text { PA }\end{array}$ & 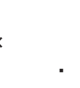 & $\cdot$ & . & 4.82 & $0.003^{a}$ & $-1.7-7.9$ \\
\hline
\end{tabular}

Table 4. Prediction of the musculoskeletal complaint-severity index (MSI) over the 12-month period following the laboratory experiment. Electromyographic (EMG) values, as percentage of peak voluntary electrical activity $\left(E M G_{\text {peak }}\right)$, in the left upper trapezius muscle during the first 40 seconds of sustained submaximal shoulder abduction ( $\left(E G_{\text {sub40 }}\right.$ ) was included as the main predictor in the multiple regression model. The MSI, the psychological complaint-severity index (PI), and physical activity (PA) over the 4 months preceding the experiment, gender, and age were entered to adjust the prediction.

\begin{tabular}{|c|c|c|c|c|c|c|}
\hline \multirow[t]{3}{*}{ Independent variables } & \multicolumn{6}{|c|}{ MSI over a 12-month period following the laboratory experiment } \\
\hline & \multicolumn{3}{|c|}{ Crude estimates } & \multicolumn{3}{|c|}{ Adjusted estimates } \\
\hline & $\beta$ & $P$ & $95 \% \mathrm{Cl}$ & $\beta$ & $\mathrm{P}$ & $95 \% \mathrm{Cl}$ \\
\hline$E M G_{\text {sub40 }}\left(\%\right.$ of $\left.E M G_{\text {peak }}\right)$ in the left trapezius during shoulder abduction & -0.01 & 0.644 & $-0.05-0.03$ & -0.01 & 0.295 & $-0.03-0.01$ \\
\hline MSI over the 4 months preceding the laboratory experiment & 0.88 & $<0.001^{a}$ & $0.7-1.1$ & 0.39 & $0.008^{\text {a }}$ & $0.1-0.7$ \\
\hline PI over the 4 months preceding the laboratory experiment & 0.99 & $<0.001^{a}$ & $0.8-1.2$ & 0.68 & $<0.001^{a}$ & $0.4-1.0$ \\
\hline Gender & 0.05 & 0.828 & $-0.4-0.5$ & . & . & $\cdot$ \\
\hline Age & -0.02 & 0.166 & $-0.1-0.01$ & . & . & . \\
\hline PA & 0.14 & 0.102 & $-0.03-0.3$ & . & . & . \\
\hline
\end{tabular}

a $\mathrm{P}<0.05, \mathrm{~N}=44$. 
predict changes in the MSI using $\mathrm{EMG}_{\text {sub } 40}$ in the trapezius as an independent variable.

\section{Discussion}

This study showed that, in a working population, a history of musculoskeletal complaints or shoulder pain predicted lower trapezius muscular activity, as measured by EMG during submaximal contraction. However, the EMG levels did not predict changes in the severity of musculoskeletal complaints over a subsequent 12 -month period. No relation was found between a history of psychological complaints and muscular activity. The complaint-severity indices were generally low, and, therefore the possibility of detecting differences in muscular activity in relation to the complaints may have been decreased.

Our study was designed to obtain an adequate measure of the complaint severity of the participants and to relate this measure to muscle responses. Because complaint severity may vary considerably in time and between body regions (22, 24), our indices were computed as averages of different areas and over 4 or 12 months. We have shown that this procedure produces a reliable measure of the MSI and PI (22). The approach may conceal associations between muscle responses and local pain; hence additional analyses were carried out without averaging across body regions. Almost the same findings were obtained with the two methods of assessing complaints.

Previous data on the relation between muscular complaints and muscular activity are inconsistent. In some of the recent literature, reduced muscular activity related to experimentally induced muscular pain or disabling clinical pain has been documented during different types of muscular work with different contraction levels (25-29). Findings of reduced muscular activity with pain were the basis for the pain-adaptation hypothesis of Lund and his co-workers (12). In a prospective study of people with whiplash injury, Nederhand and his co-workers (29) showed a lower level of isometric muscular activity in people with more extensive disability 6 months after the accident. The lower activity level was seen at all the postaccident measurement times (1, 4, 8, 12, and 24 weeks) (29). A possible explanation for reduced activity in both acute and chronic states is that musculoskeletal complaints result in an altered motor activity pattern as a response or adaptation to pain. This occurrence would require more "help" from synergistic mus- cles or from other structures of the musculoskeletal system to maintain work effort. Studies of motor control show that muscular forces are redistributed in certain situations and that trapezius muscular activity can be reduced while a static posture is maintained $(30,31)$. Our findings that musculoskeletal complaints predict reduced muscular activity in the trapezius muscle during sustained submaximal work support the hypotheses of inhibited muscular activation or altered coordination in acute and chronic pain situations $(12,13)$. It may be possible to observe the hypothesized altered pattern of muscular activity in studies of experimentally induced pain, in patients with disabling complaints, and in populations with a low level of complaint severity. Yet a low level of complaint severity may still be related to the degree of complaints, as shown for the workers in our study. A similar pattern of muscular activity was observed in the relations between complaint severity and muscular activity during the first 40 seconds and the final 5 seconds of an exhausting isometric contraction. Thus fatigue does not appear to be the cause of reduced muscular activity with a higher level of complaints.

Previous studies from our laboratory, using models of sustained isometric abduction (32), did not suggest an association between myalgia and altered muscular activity during isometric contractions. There were, however, several differences in the design between the previous and present studies. First, the present study is a prospective one, whereas Røe and her co-workers (32) used a cross-sectional design. Second, joint stability requirements differed between the studies (eg, different forearm and upper arm support during shoulder abduction). This difference may have caused different co-activation of the synergists (33). These studies may provide dissimilar information about the relation between muscular activity and pain.

Our study did not show any relation between a history of psychological complaints and the investigated muscle responses. Cross-sectional studies show that emotional and cognitive load is accompanied by muscular activity $(17,34-36)$ and that reported complaints are related to muscular activity during low-intensity mechanical load or in nonengaged musculature $(23,37,38)$. Less is known about the relationship between psychological complaints and muscular activity during a higher work intensity (eg, $25 \%$ of maximal performance as in the current study). It is possible that, during a higher work intensity, musculoskeletal pain, even of low severity, may have a greater effect on 
muscular coordination than psychological complaints of low severity. It would be interesting to investigate whether similar results occur in groups with a high severity of musculoskeletal and psychological complaints, where the individual and combined effects of each complaint can be evaluated in a regression model. In our study, we observed no statistically significant interaction between muscular activity and musculoskeletal or psychological complaints. Hence, there was no evidence of a concurrent psychogenic increase in muscular activity (eg, in relation to fear avoidance) (16).

Neither the EMG levels during the sustained contractions nor those immediately after predicted changes in the severity of health complaints in the following 12-month period. These findings do not support those of Veiersted (18), who showed that a higher activity level in the trapezius muscle during accidental machine-stops of an assembly line predicted a risk of trapezius myalgia. In our study, however, the possibility of detecting altered complaint severity in relation to muscle physiology may be limited due to a relatively small study group with low-level complaints.

Men exhibited longer endurance times than women during shoulder abduction, whereas no gender differences were found for the endurance times of wrist extension. It has been documented that women have better endurance than men when performing static contractions of low or moderate intensity (39-42). Hence the gender difference in endurance times during shoulder abduction is probably caused by the slightly higher relative force of the women.

In conclusion, musculoskeletal complaints reported during a 4-month period predicted reduced EMG activity in the trapezius muscle during submaximal muscular contractions. A history of psychological complaints was not related to muscular activity. These results may support the pain-adaptation hypothesis or the neuromuscular activation model, both of which suggest a dysfunction of synergistic muscular control. Our findings did not indicate that the level of muscular activity predicts changes in complaint severity in the subsequent 12 months.

\section{Acknowledgments}

We gratefully acknowledge the cooperation of Eva S Bakke, Astrid Bolling, Shahrooz Elka, Øystein $\mathrm{K}$ Hansen, Øyvind Hesselberg, Ada Ingvaldsen,
Birgitta Jarmark-Robertsson, Stig-Helge Larsen, Dagfinn Matre, Christine Mohn, Jens C Riis, Laila Rosenborg, Cecilie Røe, Bjørg I Selberg, Janne Schiøll, Jorid T Stuenæs, Bo Veiersted and Morten Wærsted in the project. We also thank Professor Thore Egeland for his statistical advice, Cecilie Røe for her comments on the manuscript, the workers who participated in the project, and the Norway Post.

The Research Council of Norway supported this study (grant 109176/330).

\section{References}

1. Bernard BP, ed. Musculoskeletal disorders and workplace factors: a critical review of epidemiologic evidence for workrelated musculoskeletal disorders of the neck, upper extremity and low back. Cincinnati $(\mathrm{OH})$ : National Institute for Occupational Safety and Health; 1997.

2. Punnett L, Bergqvist U. Visual display unit work and upper extremity musculoskeletal disorders: a review of epidemiological findings. Solna (Sweden): National Institute of Working Life; 1997.

3. Mense S. Neurophysiology of muscle in relation to pain. In: Værøy H, Merskey H, editors. Progress in fibromyalgia and myofascial pain. Amsterdam: Elsevier Science Publishers BV; 1993. pp 23-9.

4. Besson JM. The neurobiology of pain. Lancet 1999; 353:1610-5.

5. Loeser JD, Melzack R. Pain: an overview. Lancet 1999; 353:1607-9.

6. Graven-Nielsen T, Mense S. The peripheral apparatus of muscle pain: evidence from animal and human studies. Clin J Pain 2001;17:2-10.

7. Knardahl, S. Psychophysiological mechanisms of pain in computer work: the blood vessel-nociceptor interaction hypothesis. Work Stress 2002;16:179-89.

8. Simons DG, Mense S. Understanding and measurement of muscle tone as related to clinical muscle pain. Pain 1998; 75:1-17.

9. Travell JG, Rinzler S, Herman M. Pain and disability of the shoulder and arm. J Am Med Assoc 1942;120:417-22.

10. Schmidt RF, Kniffki K-D, Schomburg ED. Der einfluss kleinkalibriger muskelafferenzen auf dem muskeltonus. In: Bauer H, Koella WP, Struppler H, editors. Treatment of spasticity. Munchen: Verlag für angewandte Wissenschaft; 1981. pp 71-86.

11. Johansson H, Sojka P. Pathophysiological mechanisms involved in genesis and spread of muscular tension in occupational muscle pain and in chronic musculoskeletal pain syndromes: a hypothesis. Med Hypotheses 1991;35:196-203.

12. Lund JP, Donga R, Widmer CG, Stohler CS. The pain-adaptation model: a discussion of the relationship between chronic musculoskeletal pain and motor activity. Can J Physiol Pharmacol 1991;69:683-94.

13. Sterling M, Jull G, Wright A. The effect of musculoskeletal pain on motor activity and control. J Pain 2001;2:135-45.

14. Cherry NM, Meyer JD, Chen Y, Holt DL, McDonald JC. The reported incidence of work-related musculoskeletal disease 
in the UK: MOSS 1997-2000. Occup Med 2001;51:4505 .

15. Ihlebæk C, Eriksen HR, Ursin H. Prevalence of subjective health complaints (SHC) in Norway. Scand J Public Health 2002;30:20-9.

16. Linton SJ. Psychological risk factors for neck and back pain. In: Nachemson A, Jonsson E, editors. Neck and back pain: the scientific evidence of causes, diagnosis, and treatment. Philadelphia (PA): Lippincott Williams \& Wilkins; 2000. pp 57-78.

17. Wærsted M, Westgaard RH. Attention-related muscle activity in different body regions during VDU work with minimal physical activity. Ergonomics 1996;39:661-76.

18. Veiersted KB. Sustained muscle tension as a risk factor for trapezius myalgia. Int J Ind Erg 1994;14:333-9.

19. Elert JE, Rantapää-Dahlqvist SB, Henriksson-Larsen K, Lorentzen R, Gerdle BU. Muscle performance, electromyography and fibre type composition in fibromyalgia and work-related myalgia. Scand J Rheumatol 1992;21:2834.

20. Elert J, Kendall SA, Larsson B, Mansson B, Gerdle B. Chronic pain and difficulty in relaxing postural muscles in patients with fibromyalgia and chronic whiplash associated disorders. J Rheumatol 2001;28:1361-8.

21. Fredin Y, Elert J, Britschgi N, Vaher A, Gerdle B. A decreased ability to relax between repetitive muscle contractions in patients with chronic symptoms after whiplash trauma of the neck. J Musculoskel Pain 1997;5:55-70.

22. Steingrímsdóttir ÓA, Vøllestad NK, Røe C, Knardahl S. Variation in reporting of pain and other subjective health complaints in a working population and limitations of single sample measurements. Pain 2004;110:130-9.

23. Steingrímsdóttir ÓA, Vøllestad NK, Knardahl S. A prospective study of the relation between musculoskeletal or psychological complaints and muscular responses to standardized cognitive and motor tasks in a working population. Eur $\mathrm{J}$ Pain. In press.

24. Von Korff M, Saunders K. The course of back pain in primary care. Spine 1996;21:2833-7.

25. Birch L, Graven-Nielsen T, Christensen H, Arendt-Nielsen L. Experimental muscle pain modulates muscle activity and work performance differently during high and low precision use of a computer mouse. Eur J Appl Physiol 2000;83:492-8.

26. Arima T, Arendt-Nielsen L, Svensson P. Effect of jaw muscle pain and soreness evoked by capsaicin before sleep on orofacial motor activity during sleep. J Orofac Pain 2001;15:245-56.

27. Svensson P, Graven-Nielsen T. Craniofacial muscle pain: review of mechanisms and clinical manifestations. J Orofac Pain 2001;15:117-45.

28. Ng JK-F, Richardson CA, Parnianpour M, Kippers V. EMG activity of trunk muscles and torque output during isometric axial rotation exertion: a comparison between back pain patients and matched controls. J Orthop Res 2002;20:11221.

29. Nederhand MJ, Hermens HJ, Ijzerman MJ, Turk DC, Zilvold G. Chronic neck pain disability due to an acute whiplash injury. Pain 2003;102:63-71.

30. Palmerud G, Kadefors R, Sporrong H, Järvholm U, Herberts $\mathrm{P}$, Högfors $\mathrm{C}$, et al. Voluntary redistribution of muscle activity in human shoulder muscles. Ergonomics 1995;38:80615 .

31. Palmerud G, Sporrong H, Herberts P, Kadefors R. Consequences of trapezius relaxation on the distribution of shoulder muscle forces: an electromyographic study. J Electromyogr Kinesiol 1998;8:185-193.

32. Røe C, Knardahl S, Vøllestad NK. Muscle activation during isometric contractions in workers with unilateral shoulder myalgia. J Musculoskeletal Pain 2000;8:57-73.

33. Solomonow M., Krogsgaard M. Sensorimotor control of knee stability: a review. Scand J Med Sci Sports 2001;11:64-80.

34. Jacobson E. Action currents from muscular contractions during conscious processes. Science 1927;66:403.

35. Wærsted M, Bjørklund RA, Westgaard RH. Shoulder muscle tension induced by two VDU-based tasks of different complexity. Ergonomics 1991;34:137-50.

36. Wærsted M, Bjørklund RA, Westgaard RH. The effect of motivation on shoulder-muscle tension in attention-demanding tasks. Ergonomics 1994;37:363-76.

37. Hovanitz CA, Filippides M, Lindsay D, Scheff J. Muscle tension and physiologic hyperarousal, performance, and state affectivity: assessing the independence of effects in frequent headache and depression. Appl Psychophysiol Biofeedback 2002;27:29-44.

38. Van Galen GP, Müller ML, Meulenbroek RG, van Gemmert AW. Forearm EMG response activity during motor performance in individuals prone to increased stress reactivity. Am J Ind Med 2002;41:406-19.

39. Maughan RJ, Harmon M, Leiper JB, Sale D, Delman A. Endurance capacity of untrained males and females in isometric and dynamic muscular contractions. Eur J Appl Physiol Occup Physiol 1986;55:395-400.

40. Sato H, Ohashi J. Sex differences in static muscular endurance. J Hum Ergol (Tokyo) 1989;18:53-60.

41. West W, Hicks A, Clements L, Dowling J. The relationship between voluntary electromyogram, endurance time and intensity of effort in isometric handgrip exercise. Eur J Appl Physiol Occup Physiol 1995;71:301-5.

42. Fulco CS, Rock Pb, Muza SR, Lammi E, Cymerman A, Butterfield G, et al. Slower fatigue and faster recovery of the adductor pollicis muscle in women matched for strength with men. Acta Physiol Scand 1999;167:233-9.

Received for publication: 17 December 2003 